\title{
Evaluación física, química y microbiológica del ensilaje de yuca con caupí y cultivo microbiano
}

\section{(Physical, chemical and microbiological evaluation of cassava silage with cowpea and microbial culture)}

\author{
JE Miranda-Yuquilema ${ }^{1}$, A Marin-Cárdenas², M González-Pérez³ ${ }^{3}$ D Sánchez-Macías ${ }^{4}$
}

\begin{abstract}
Resumen:
El objetivo de este trabajo fue evaluar los cambios físicos, químicos, microbiológicos, fibrosos y del ácido láctico del ensilaje de yuca con caupí y cultivo microbiano. Se utilizaron $50 \mathrm{~kg}$ de yuca integral (raíz, tallo, hojas), $20 \mathrm{~kg}$ de harina de caupí y $4 \mathrm{~L}$ de cultivo microbiano que contenía Lactobacillus acidophillus, Streptococcus thermophillus y Klyumyces fragilis (L-4 UCLV). Las variantes evaluadas fueron: yuca integral (Y100), yuca integral más $20 \%$ caupí $(Y+C)$, yuca integral más $20 \%$ caupí y $8 \%$ biopreparado $(\mathrm{Y}+\mathrm{C}+\mathrm{BP})$. El material ensilado se conservó en frascos de cristal estériles con boca ancha. Se utilizó un diseño completamente aleatorio. Se evaluaron las características fisicoquímicas, fibrosas, microbiológicas y de ácido láctico. Durante 18 meses, los contenidos de materia seca, proteína cruda y verdadera, ácido láctico y la viabilidad microbiana fue mayor $(\mathrm{P}<0.05)$ en el tratamiento $\mathrm{Y}+\mathrm{C}+\mathrm{BP}$ a partir de los dos meses hasta el final. La fibrosidad y el $\mathrm{pH}$ del ensilaje fue menor $(P<0.05)$ al final del estudio en el tratamiento $\mathrm{Y}+\mathrm{C}+\mathrm{BP}$. Se concluye que con la inclusión del cultivo microbiano en el ensilaje de yuca con caupí se logra conservar las propiedades fisicoquímicas y microbiológicas por 18 meses; asimismo, los valores de la materia seca, proteína cruda y verdadera y ácido láctico se mejoran.
\end{abstract}

Palabras clave: ensilado; cultivo microbiano; caupí; yuca.

\begin{abstract}
:
The aim of this work was to evaluate the physical, microbiological, fibrous and lactic acid changes of cassava silage with cowpea used on biological mix. Fifty kg whole cassava (root, stem and leaves), $20 \mathrm{~kg}$ of cowpea flour and $4 \mathrm{~L}$ of microbial culture containing Lactobacillus acidophillus, Streptococcus thermophillus and Klyumyces fragilis (L-4 UCLV) were used. The evaluated treatments were: Y100integral cassava (Y100), integral cassava more $20 \%$ cowpea $(Y+C)$, and integral cassava more $20 \%$ cowpeas and $8 \%$ of microbial culture $(\mathrm{Y}+\mathrm{C}+\mathrm{BP})$. The ensiled material was preserved in sterile glass bottles with wide mouth. A completely randomized design was used. The physicochemical, microbiological, fibrous and lactic acid characteristics were evaluated. For 18 months, the dry matter, crude and true protein, lactic acid contents and the microbial viability was higher $(\mathrm{P}<0.05)$ in $\mathrm{Y}+\mathrm{C}+\mathrm{BP}$ treatments from 2 to 18 months. The fibrosity and the $\mathrm{pH}$ of the silage was also lower $(\mathrm{P}<0.05)$ at the end of the study in this treatment $\mathrm{Y}+\mathrm{C}+\mathrm{BP}$. It is concluded that with the inclusion of the microbial mix in the cassava silage with cowpea, it is possible to preserve the physicochemical and microbiological properties for 18 months, as well as the values of dry matter, crude and true protein, and lactic acid contents were improved.
\end{abstract}

Keywords: silage; microbial culture; cassava; cowpea.

\footnotetext{
1 Instituto de Fomento a Talento Humano, SENESCYT, Ecuador (efra_miranda@outlook.com)

2 Universidad Central "Marta Abreu" de Las Villas, Santa Clara - Cuba

${ }^{3}$ Centro de Investigación Agropecuarias (CIAP-UCLV), Santa Clara - Cuba

4 Universidad Nacional de Chimborazo, Riobamba - Ecuador
} 


\section{Introducción}

La producción y conservación de los tubérculos, granos, frutales y forrajes es clave en muchas regiones del mundo, sobre todo en los países en vía de desarrollo, para paliar los desafíos actuales que atraviesa el planeta. En tal sentido, se hace apremiante la búsqueda de soluciones y alternativas que logren dar respuesta a las necesidades básicas en la producción animal (Hoffman et al., 2011). Un método económico y de fácil acceso es el ensilaje, el cual permite conservar productos de rápido deterioro. A su vez, permite aumentar los valores biológicos en los productos obtenidos mediante estas técnicas (Danelon et al., 2010). En las últimas décadas se ha dado lugar el desarrollo de ensilaje con fines de obtener productos en mayores cantidades y con alto valor biológico durante la conservación (Fernández et al., 2013) al reducir la pérdida de nutrientes por rápido deterioro y compensar los costos de producción (Miranda et al., 2014; Ali et al., 2014). El aumento continuo de costos de los alimentos balanceados para animales, en la mayoría de los casos llegando a superar el $80 \%$ del costo total de producción (Heguy et al., 2016), ha limitado su uso y minimizado las utilidades para el pequeño y mediano productor.

No obstante, el uso de la planta entera de yuca (hoja, tallo y raíz) mezclada con harina de caupí, a lo cual se adicionan cultivos microbiológicos, pudiera resultar eficiente y económico para obtener ensilajes para la alimentación animal. En este sentido, se ha encontrado en la literatura estudios que utilizan técnicas de ensilaje como un medio idóneo para conservar el contenido nutritivo de los forrajes, tubérculos, granos y frutos (alfalfa, hojas de maíz, sorgo, plátano, yuca, patatas, frijoles forrajeros, pastos, entre otros) (Heguy et al., 2012; Spanghero et al., 2015).

A base de lo anterior, los alimentos alternativos han sido evaluados y utilizados para diferentes especies animales con resultados positivos. Un ejemplo de esto es el aumento de peso y ganancia media diaria de peso de cerdos en la etapa ceba (Kung et al., 2015). En las vacas y cabras lecheras se ha visto mejorar la producción de leche (Danelon et al., 2010; Guglielmo et al., 2015). Unido a esto, Ali et al. (2014), al incluir 50\% de ensilaje con enzimas celulolíticas en pruebas "in situ", observaron mejorar la degradación de alimentos fibrosos. Asimismo, Miranda et al. (2014) reportaron extender el tiempo de conservación sin afectar al contenido nutritivo y con reducción de las partes fibrosas de la yuca al inocular cultivo mixto de bacterias ácido lácticas y levaduras. De igual manera, Hoffman et al. (2011), Dadvar et al. (2013) y Lyncha et al. (2014), con el uso de un cultivo mixto se lograron controlar el crecimiento de microorganismos toxigénicos como clostridium, $S$. aureus, $C$. botulinum, Salmonella sp. y Listeria monocytogenes, así como de los microorganismos asociados al deterioro directo del alimento. Si se tiene en cuenta todo lo anterior, el presente estudio tuvo como objetivo evaluar los cambios físicos, químicos, microbiológicos, fibrosos y ácido láctico del ensilaje de yuca con caupí al emplear cultivo microbiano, durante 18 meses de conservación.

\section{Materiales y Métodos}

Área de estudio: el estudio se realizó en el laboratorio de bromatología, Centro de Investigaciones Agropecuarias (CIAP) y laboratorio de microbiología, Facultad de Ciencias Agropecuarias, Universidad Central "Marta Abreu" de Las Villas, Santa Clara, Cuba.

Material empleado: se emplearon $50 \mathrm{~kg}$ de la planta entera (hoja, tallo y raíz) de yuca (Manihot esculenta crantz) fresca, con las siguientes características: $43.43 \%$ de materia seca (MS), $4.5 \%$ de proteína cruda (PC) y 0,9\% ceniza $(\mathrm{Cz}) ; 20 \mathrm{~kg}$ harina de caupí (Vigna unguiculata) que contenía $85 \%$ MS, 26.1\% PC, $1.0 \%$ extracto etéreo (EE), y 3.2 $\mathrm{Cz}$, y $5 \mathrm{~L}$ de preparado microbiano.

Selección, activación de las cepas y obtención del preparado microbiano: las cepas seleccionadas para la obtención del preparado microbiano fueron Lactobacillus 
acidophillus, Streptococcus thermophillus y Kluyveromyces fragilis (L-4 UCLV) proveniente del banco de microorganismos de la Universidad Central "Marta Abreu" de Las Villas. Las cepas en formato biomasa fueron activadas en $250 \mathrm{~mL}$ leche de soya ácida a $37^{\circ} \mathrm{C}$ por 24 h en una estufa con zaranda (Nkubationshaube TH 15, Alemania) a $60 \mathrm{rpm}$. Una vez activados los microorganismos, se procedió a obtener el preparado microbiano. Se inocularon $150 \mathrm{mg}$ (Balanza Analítica Radwag AS 220/C/2, Suiza) de biomasa anteriormente descrita en una mezcla de $2.5 \mathrm{~L}$ de yogur de soya y $600 \mathrm{~g}$ de melaza de caña de azúcar a $34 \pm 2^{\circ} \mathrm{C}$. La mezcla se incubó a $37^{\circ} \mathrm{C}$ durante $24 \mathrm{~h}$. Finalmente se caracterizó de acuerdo con Miranda et al. (2014). El preparado microbiano previamente obtenido contenía $13.35 \%$ MS, $3.5 \%$ de proteína bruta (PB), $3.9 \mathrm{pH}$ y $9 \times 10^{8}$ y $9.1 \times 10^{7}$ (bacterias y levaduras) UFC/mL de concentración microbiana.

Obtención de las variantes: la yuca entera (raíz, tallo, hojas) fue molida utilizando un molino martillo (modelo 301011, España), hasta obtener un tamaño de partículas entre 5-8 mm. Luego, según el tratamiento detallado en la Tabla 1, se mezcló con la harina de caupí, y posteriormente se procedió a inocular el preparado microbiano, en el caso del tratamiento con cultivo microbiano. Todos los tratamientos fueron conservados en frascos de cristal estériles de boca ancha con capacidad de $1 \mathrm{~kg}$, y a una temperatura ambiente de $12 \pm 2^{\circ} \mathrm{C}$.

Tabla 1. Los tratamientos empleados en el estudio.

\begin{tabular}{cc}
\hline Tratamientos & Variantes \\
\hline Y100 & Yuca integral (raíz + tallo + hoja) \\
Y $+\mathrm{C}$ & Yuca integral más 20\% harina de caupí \\
Y+C+BP & Yuca integral más 20\% harina de caupí $+8 \%$ preparado microbiano \\
\hline \multicolumn{2}{c}{ Los tratamientos se realizaron por triplicado en diferentes días. }
\end{tabular}

Caracterización, física y química: el color se evaluó mediante código HTML de acuerdo con Miranda et al. (2017). El aroma, sabor y textura se evaluaron por los sentidos sensoriales del investigador mediante la metodología descrita por Acevedo et al. (2009). La determinación de PC y proteína verdadera (PV) se realizó de acuerdo con Dadvar et al. (2015). Los contenidos de MS, Cz, EE, fibra acida detergente (FAD), lignina y celulosa, se determinaron mediante los métodos de AOAC (2012). El pH se medió con un pHmetro (HANNA® *H 110, USA), calibrado a 4, 7 y 10.

Análisis microbiológico: a $25 \mathrm{~g}$ de cada tratamiento se le añadieron $50 \mathrm{~mL}$ de suero fisiológico y se incubó a $37^{\circ} \mathrm{C}$ por $24 \mathrm{~h}$. A continuación, las muestras se centrifugaron las muestras (centrífuga BD DYNAC ${ }^{\mathrm{TM}} \mathrm{III}$ ) a $600 \mathrm{rpm}$ por 5 minutos. La determinación de ácido láctico se realizó según la técnica "Conway" descrita por Conway (1957). Para el análisis de viabilidad, los tratamientos fueron preparados en las diferentes concentraciones hasta la escala 0,5 del esquema McFarland para la viabilidad. Posteriormente, se cultivaron las muestras y se realizó el recuento celular de número de unidades formadoras de colonias (UFC), según la metodología descrita por Miranda et al. (2017).

Análisis estadístico: la comparación de medias de los tratamientos se realizó mediante la prueba de Duncan (1955), y estos fueron analizados usando el software Statgraphic Plus versión 51.1.

\section{Resultados}

En la Figura 1 se observa la caracterización organoléptica del ensilaje de yuca (Y100), yuca con caupí $(\mathrm{Y}+\mathrm{C})$ y de yuca más caupí y cultivo microbiano $(\mathrm{Y}+\mathrm{C}+\mathrm{BP})$. El aroma y el sabor fueron de ácido a ácido dulzón; color verde lechoso a marrón; la textura fue semisólida al momento de ensilar para todas las variantes. Dichas características no 
variaron hasta dos meses de conservado. En la medición a los 12 y 18 meses, se observó diferencias entre los tratamientos, pero el de mejor comportamiento fue la variante $\mathrm{Y}+\mathrm{C}+\mathrm{BP}$.

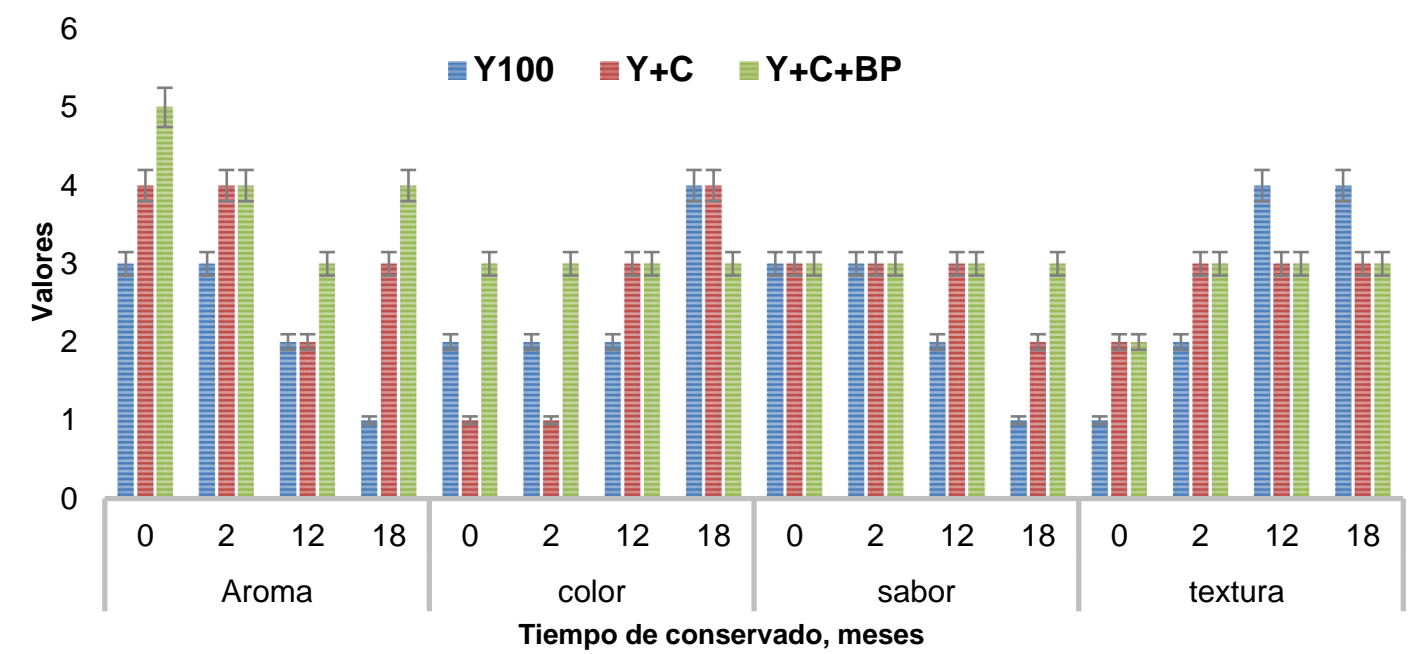

Figura 1. Características organolépticas de los ensilajes. Y100. Yuca integral (hoja, talla. raíz). Y+C. Yuca más $20 \%$ harina de caupí. Y+C+BP. Yuca más $20 \%$ harina de

En la Tabla 2 se resumen los valores medios de la composición química básica de los ensilajes. Al momento del ensilar, la MS, PC y PV fue menor $(\mathrm{P}<0.05)$ en el tratamiento Y100 en comparación con los otros tratamientos, sin variación entre estos dos últimos. Además, la $\mathrm{Cz}$ y EE no difirió entre variantes. A los 2, 12 y 18 meses, el contenido de MS, $\mathrm{PC}$ y $\mathrm{PV}$ fue mayor $(\mathrm{P}>0.05)$ en $\mathrm{Y}+\mathrm{C}+\mathrm{BP}$ que en los otros tratamientos. En cuanto al $\mathrm{EE}$, fue menor $(\mathrm{P}<0.05)$ en $\mathrm{Y}+\mathrm{C}+\mathrm{BP}$ frente a $\mathrm{Y} 100 \mathrm{y} \mathrm{Y}+\mathrm{C}$ a los 12 y 18 meses de conservado. Los valores de $\mathrm{Cz}$ no defirieron entre tratamientos durante todo el estudio.

Tabla 2. Característica química del ensilado de yuca con caupí, al emplear preparado microbiano durante la conservación.

\begin{tabular}{|c|c|c|c|c|c|c|}
\hline \multirow{2}{*}{$\begin{array}{c}\text { Tiempo, } \\
\text { meses }\end{array}$} & \multirow{2}{*}{ Indicadores } & \multicolumn{3}{|c|}{ Tratamientos } & \multirow{2}{*}{ SEM } & \multirow{2}{*}{ P-value } \\
\hline & & Y100 & $\mathrm{Y}+\mathrm{C}$ & $\mathrm{Y}+\mathrm{C}+\mathrm{BP}$ & & \\
\hline \multirow{5}{*}{0} & Materia seca \% $(\mathrm{m} / \mathrm{m})$ & $25.25^{b}$ & $30.32^{a}$ & $30.57^{a}$ & 0.07 & 0.0025 \\
\hline & Proteína cruda \%, (m/m) & $11.12^{\mathrm{b}}$ & $12.34^{\mathrm{a}}$ & $12.12^{\mathrm{a}}$ & 0,01 & 0.0056 \\
\hline & Proteína verdadera \% $(\mathrm{m} / \mathrm{m})$ & $7.11^{\mathrm{b}}$ & $8.25^{a}$ & $8.14^{a}$ & 0,01 & 0.0125 \\
\hline & Extracto etéreo \%, (v/v) & 2.21 & 2.28 & 2.22 & 0.02 & 0.2532 \\
\hline & Ceniza \%, (m/v) & 3.92 & 3.73 & 3.68 & 0.03 & 0.2307 \\
\hline \multirow{5}{*}{2} & Materia seca \% $(\mathrm{m} / \mathrm{m})$ & $29.75^{c}$ & $33.22^{b}$ & $34.17^{a}$ & 0.03 & 0.0042 \\
\hline & Proteína cruda \%, (m/m) & $11.12^{\mathrm{c}}$ & $13.14^{\mathrm{b}}$ & $14.21^{\mathrm{a}}$ & 0.11 & 0.0271 \\
\hline & Proteína verdadera \% (m/m) & $7.81^{\mathrm{c}}$ & $8.73^{b}$ & $9.94^{a}$ & 0.06 & 0.0125 \\
\hline & Extracto etéreo \%, (v/v) & 2.18 & 2.51 & 2.12 & 0.04 & 0.6495 \\
\hline & Ceniza \%, (m/v) & 3.52 & 3.63 & 3.44 & 0.87 & 0.1389 \\
\hline \multirow{5}{*}{12} & Materia seca $\%(\mathrm{~m} / \mathrm{m})$ & $32.25^{c}$ & $37.12^{b}$ & $39.34^{a}$ & 0,13 & $<.0001$ \\
\hline & Proteína cruda \%, (m/m) & $10.23^{c}$ & $12.84^{\mathrm{b}}$ & $13.03^{a}$ & 0.10 & 0.0028 \\
\hline & Proteína verdadera \% $(\mathrm{m} / \mathrm{m})$ & $7.22^{\mathrm{c}}$ & $9.22^{b}$ & $10.11^{\mathrm{a}}$ & 0,11 & 0.0125 \\
\hline & Extracto etéreo \%, $(\mathrm{v} / \mathrm{v})$ & $2.43^{a}$ & $2.51^{\mathrm{a}}$ & $1.89^{\mathrm{b}}$ & 0.01 & 0.0054 \\
\hline & Ceniza \%, (m/v) & 3.92 & 3.73 & 3.77 & 0,13 & 0.0675 \\
\hline \multirow{5}{*}{18} & Materia seca $\%(\mathrm{~m} / \mathrm{m})$ & $22.61^{\mathrm{c}}$ & $35.14^{b}$ & $39.58^{a}$ & 0.10 & $<.0001$ \\
\hline & Proteína cruda \%, (m/m) & $5.12^{\mathrm{c}}$ & $11.07^{\mathrm{b}}$ & $13.33^{a}$ & 0,11 & $<.0001$ \\
\hline & Proteína verdadera \% $(\mathrm{m} / \mathrm{m})$ & $2.32^{\mathrm{c}}$ & $7.65^{b}$ & $9.64^{a}$ & 0.01 & 0.0012 \\
\hline & Extracto etéreo \%, (v/v) & $2.06^{b}$ & $2.14^{\mathrm{a}}$ & $1.32^{\mathrm{a}}$ & 0.11 & 0.0125 \\
\hline & Ceniza \%, $(\mathrm{m} / \mathrm{v})$ & 3.12 & 3.03 & 3.23 & 0,89 & 0.2030 \\
\hline
\end{tabular}

a,b,c letras desiguales en la misma fila, las medias difieren a $\mathrm{P}<0.05$ (Duncan, 1955). Y100. Yuca (hoja, talla, raíz). Y+C, Yuca más $20 \%$ harina de caupí. Y+C+BP. Yuca más $20 \%$ harina de caupí y $8 \%$ preparado microbiano. $\mathbf{m} / \mathbf{m}$, masa/masa. $\mathbf{v} / \mathbf{v}$, volumen/volumen. $\mathbf{m} / \mathbf{v}$, masa/volumen. 
En la Tabla 3 se presentan los valores medios de FAD, lignina y celulosa. Estos no variaron entre tratamientos al momento de ensilar. Tras dos meses de conservado, la FAD fue menor $(P=0.0021)$ en $Y+C+B P$, en tanto que la lignina y celulosa no variaron entre $Y 100$ y $Y+C$. A los 12 meses después de ensilar las partes fibrosas se redujeron en todas las variantes, $y$ fue menor $(P<0.05)$ en el $Y+C+B P$ frente a $Y 100$ y $Y+C$. La lignina no mostró variación entre $\mathrm{Y} 100$ y $\mathrm{Y}+\mathrm{C}$, en tanto que la celulosa no difirió entre tratamientos. En la medición realizada a los 18 meses después de ensilar fue menor $(P<0.05)$ en $Y+C+B P$ frente a $\mathrm{Y} 100$ y $\mathrm{Y}+\mathrm{C}$, sin diferencias entre estos últimos

Tabla 3. Característica de la fibra del ensilado de yuca con caupí, al emplear preparado microbiano en las diferentes etapas de conservación

\begin{tabular}{|c|c|c|c|c|c|c|}
\hline \multirow{2}{*}{ Tiempo, meses } & \multirow{2}{*}{ Indicadores } & \multicolumn{3}{|c|}{ Tratamientos } & \multirow{2}{*}{ SEM } & \multirow{2}{*}{ P-value } \\
\hline & & Y100 & $\mathrm{Y}+\mathrm{C}$ & $\mathrm{Y}+\mathrm{C}+\mathrm{BP}$ & & \\
\hline \multirow{3}{*}{0} & FAD \%, (m/v) & 11.18 & 11.28 & 11.22 & 0.01 & 0.8401 \\
\hline & Lignina $\%(\mathrm{~m} / \mathrm{v})$ & 4.78 & 4.72 & 4.88 & 0.01 & 0.5721 \\
\hline & Celulosa \% (m/v) & 0.65 & 0.71 & 0.62 & 0.06 & 0.0642 \\
\hline \multirow{3}{*}{2} & FAD \%, (v/v) & $10.98^{a}$ & $10.44^{b}$ & $9.62^{c}$ & 0.04 & 0.0021 \\
\hline & Lignina \% $(\mathrm{m} / \mathrm{v})$ & $4.52^{a}$ & $4.53^{a}$ & $4.01^{b}$ & 0.12 & 0.0125 \\
\hline & Celulosa \% (m/v) & $0.61^{a}$ & $0.62^{\mathrm{a}}$ & $0.49^{b}$ & 0.04 & 0.0053 \\
\hline \multirow{3}{*}{12} & FAD \%, (v/v) & $10.93^{a}$ & $9.98^{b}$ & $8.92^{c}$ & 0.01 & 0.0271 \\
\hline & Lignina \% $(\mathrm{m} / \mathrm{v})$ & $4.15^{\mathrm{a}}$ & $4.02^{\mathrm{a}}$ & $3.21^{b}$ & 0.01 & 0.0125 \\
\hline & Celulosa \% (m/v) & 0.55 & 0.51 & 0.29 & 0.04 & 0.0578 \\
\hline \multirow{3}{*}{18} & FAD \%, (v/v) & $9.78^{a}$ & $9.88^{a}$ & $6.22^{b}$ & 0.01 & $<.0001$ \\
\hline & Lignina \% (m/v) & $3.89^{a}$ & $3.43^{a}$ & $1.73^{b}$ & 0.03 & 0.0025 \\
\hline & Celulosa \% (m/v) & $0.51^{\mathrm{a}}$ & $0.42^{\mathrm{a}}$ & $0.11^{\mathrm{b}}$ & 0.02 & 0.0125 \\
\hline
\end{tabular}

En la Tabla 4 se observa la viabilidad microbiana y los valores de $\mathrm{pH}$ del ensilaje. Al momento de ensilar los valores de $\mathrm{pH}$ no mostró diferencia entre tratamientos, sin embargo, a partir de dos meses hasta el final del estudio fue menor $(P<0.05)$ en el $Y+C+B P$ frente a los demás tratamientos. En cuanto a la viabilidad microbiana fue mayor $(P<0.05)$ en el $\mathrm{Y}+\mathrm{C}+\mathrm{BP}$ con respecto al $\mathrm{Y} 100$ y $\mathrm{Y}+\mathrm{C}$ a partir de dos meses hasta los 18 meses de conservado.

Tabla 4. Característica microbiológica y los valores de pH del ensilaje de yuca con caupí al incluir preparado microbiano.

\begin{tabular}{|c|c|c|c|c|c|c|}
\hline \multirow{2}{*}{ Tiempo, meses } & \multirow{2}{*}{ Indicadores } & \multicolumn{3}{|c|}{ Tratamientos } & \multirow{2}{*}{ SEM } & \multirow{2}{*}{ P-value } \\
\hline & & Y100 & $\mathrm{Y}+\mathrm{C}$ & $\mathrm{Y}+\mathrm{C}+\mathrm{BP}$ & & \\
\hline \multirow{2}{*}{0} & Viabilidad \% (v/v) & 94 & 94 & 94 & 0.01 & 0.8431 \\
\hline & $\mathrm{pH}$ & 4.42 & 4.46 & 4.37 & 0.12 & 0.2542 \\
\hline \multirow{2}{*}{2} & Viabilidad \% (v/v) & $93^{b}$ & $93^{b}$ & $94^{a}$ & 0.01 & 0.0210 \\
\hline & $\mathrm{pH}$ & 4.12 & 4.15 & 3.98 & 0.12 & 0.5214 \\
\hline \multirow[b]{2}{*}{12} & Viabilidad \%, (v/v) & $89^{b}$ & $90^{\mathrm{b}}$ & $94^{a}$ & 0.11 & $<.0001$ \\
\hline & $\mathrm{pH}$ & $5.53^{a}$ & $4.53^{b}$ & $3.97^{c}$ & 0.03 & 0.0015 \\
\hline \multirow[b]{2}{*}{18} & Viabilidad \%, (v/v) & $81^{c}$ & $89^{b}$ & $93^{a}$ & 0.02 & 0.0025 \\
\hline & $\mathrm{pH}$ & $6.83^{a}$ & $5.81^{b}$ & $3.97^{c}$ & 0.11 & $<.0001$ \\
\hline
\end{tabular}

En la Figura 2 se observa la concentración de ácido láctico en el ensilaje de yuca con caupí al inocular preparado microbiano. A partir de los 2 y hasta los 18 meses, los valores de ácido láctico fue mayor $(\mathrm{P}<0.05)$ en el tratamiento que contenida cultivo microbiano $(\mathrm{Y}+\mathrm{C}+\mathrm{BP})$ en comparación con $\mathrm{Y} 100$ y $\mathrm{Y}+\mathrm{C}$. 


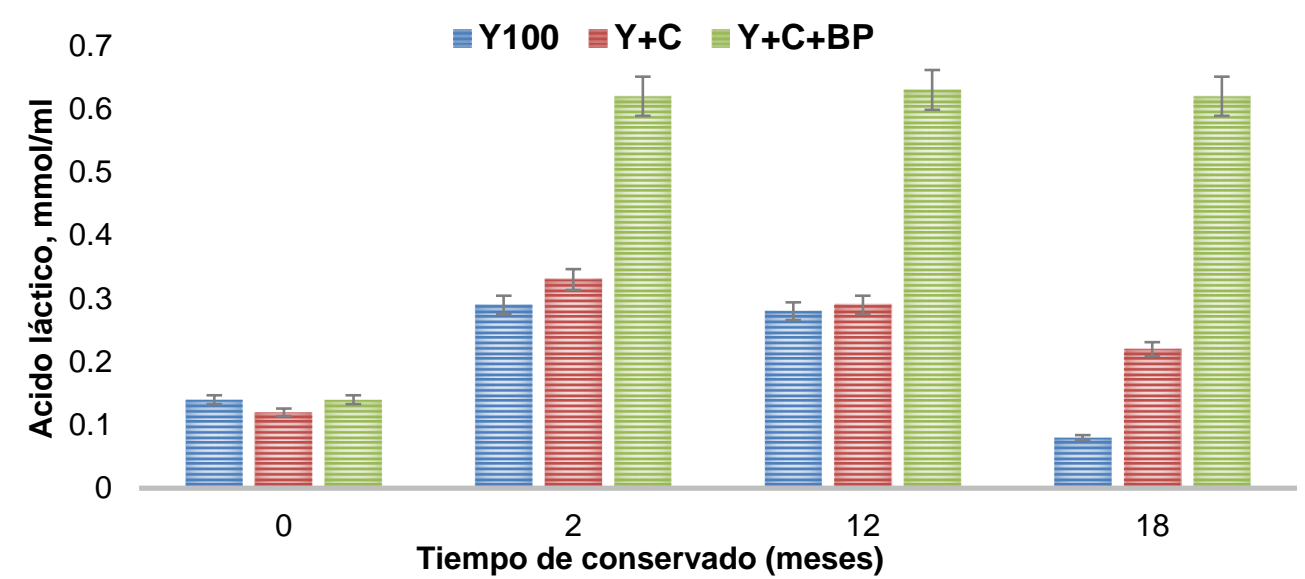

Figura 2. Comportamiento de ácido láctico del ensilaje durante 18 meses. Y100. Yuca (hoja, talla, raíz). Y+C, Yuca más $20 \%$ harina de caupí. Y+C+BP, Yuca más $20 \%$ harina de caupí y $8 \%$ preparado microbiano.

\section{Discusión}

Los resultados en cuanto a las características organolépticas en las variantes ensiladas del presente estudio (Figura 1), podrían estar relacionados con las propiedades funcionales que son reflejados atributos intrínsecos de la composición física, así como las posibles interacciones con otros componentes (Ke et al., 2015). En tal sentido, Gugglielmo et al. (2015) reportaron la repercusión de la materia prima utilizada en la característica física de los ensilados. Por su parte, Danelon et al. (2010) observaron efecto ambiental, calidad del material y el valor nutritivo en la característica física del producto final, aunque este depende fundamentalmente de la materia prima, especies y la variedad que se emplea. En tal sentido, Fernández et al. (2013) presumen que el empleo de los microorganismos influye positivamente en las características físicas posiblemente por la acción fermentativa de los microorganismos anaerobios en las primeras 24 horas después de ensilar (Miranda et al., 2014). Los resultados obtenidos en el presente estudio se asemejan con los reportados por Fernández et al. (2013). Por su parte, Lyncha et al. (2014) reportaron pH menor a 4.70 al emplear enzimas fibrolíticas en el ensilaje con alfalfa. Unido a esto, en estudio previo, Miranda et al. (2014) reportaron pH inferior a 4.23 al ensilar yuca más caupí y BIOPRANAL. Sin embargo, Ke et al. (2015) observaron pH con valores superiores a 5.85 y esto repercutía en las características físicas de los ensilajes.

Los resultados en cuanto a los parámetros químicos (Tabla 2) posiblemente fueron debidos al contenido de la materia prima y los microorganismos empleados para obtener ensilajes. Spanhero et al. (2015) observaron la influencia de materia prima sobre la calidad del ensilaje obtenido. Con referencia a lo anterior, Fernández et al. (2013) reportaron, efecto de la materia seca, contenido de compuestos nitrogenados, pérdidas de azúcares, cantidad de minerales y la capacidad de producir ácidos orgánicos del material a ensilar, sobre las características bromatológicas. Por su parte, Kung et al. (2015) observaron proteólisis durante el ensilaje, causado principalmente por los clostridium y esto aumentó sustancialmente los valores de $\mathrm{pH}$ hasta lograr la degradación el material ensilado. Mayor porciento de MS obtenido en el estudio podría estar relacionado con la evaporación de agua, unido al mayor desarrollo de microorganismos anaerobios empleado durante el ensilaje, similares valores fueron reportados por Miranda et al. (2014) al incluir BIOPRANAL en el ensilaje de yuca con caupí. Por su parte, Ke et al. (2015) observaron un leve aumento en la composición nutritiva al incluir bacterias ácido lácticas en el ensilaje de hojas de maíz. Un ligero aumento en la composición nutritiva también fue reportado por Ali et al. (2014) en pruebas realizadas "in vitro". Según el estudio de Hoffman et al. (2011) mejoraron el contenido de PC y PV al inocular biopreparados en el ensilaje de maíz con alta humedad, 
Sin embargo, Guglielmo et al. (2015) no observaron variación al ensilar sorgo con maíz. A diferencia de lo anterior Danelon et al. (2010) al emplear concentraciones energéticas en alfalfa mejoraron los valores de PC, PV y EE. No obstante, dichos parámetros se encuentran por debajo de los obtenidos en el presente estudio.

Asimismo, los valores obtenidos en cuanto a FAD, lignina y celulosa, son inferiores a los reportados por Heguy et al. (2010). Sin embargo, se asemejan con los reportados por, del Río et al. (2010). Por su parte Lyncha et al. (2004) reportaron efecto de enzimas fibrolíticas sobre la degradación de las partes fibrosas del ensilaje. Del mismo modo, Kung et al. (2015) redujeron la cantidad de lignina en el ensilaje de sorgo con maíz. Por su parte Der Bedrosian et al. (2012) reportaron la reducción de los niveles de la celulosa y lignina al ensilar maíz planta entera. De modo similar Dadvar et al. (2015) observaron al fermentar pulpa de limón con S. cerevisiae. Por su parte, Heguy et al. (2016) mejoraron los índices nutritivos y redujeron los niveles de lignina al emplear enzimas. Mientras que Danelon et al. (2010) observaron la reducción en las fracciones celulósica y esta diferencia podría estar relacionada con el contenido de almidón y microorganismos con actividad fermentativa. Estudios previos fueron realizados por del Río et al. (2010) y Hoffman et al. (2011) al determinar los contenidos de lignina, celulosa y hemicelulosa, antes y después de ensilar con preparados microbianos, pero no informaron los valores obtenidos en dichos estudios.

Sin embargo, existen reportes sobre la asimilación de lactato en algunas especies de levaduras, lo que ayuda a mejorar el crecimiento de los microrganismos anaerobios en el ensilaje (Sourav y Arijit, 2010; Ali et al., 2014). La viabilidad microbiana obtenida en el presente estudio pone de manifiesto que los microorganismos empleados se mantuvieron activos durante 18 meses y esto podría ser principalmente por la acción acidificante generada por las bacterias ácido lácticas (Dadvar et al., 2015); el mismo comportamiento fue observada por Heguy et al. (2010) al emplear biopreparados. Asimismo, Hoffman et al. (2011) reportaron ligero aumento en la viabilidad microbiana en los ensilados al incluir $10 \%$ de yogur natural. Unido a esto, Ke et al. (2015) y Spanghero et al. (2015) observaron también mejorar la producción de microorganismos benéficos en los ensilajes al emplear medios biológicos. Por su parte, (Guglielmo et al., 2015) reportaron mayor crecimiento de levaduras ligninolíticas en los ensilajes de maíz con alto grado de humedad. Sin embargo, en los estudios realizados por del Río et al. (2010), Ali et al. (2014) y Heguy et al. (2016) se vio que con el empleo de las enzimas en los ensilajes desarrollados con tallos de tabaco, algodón, girasol y paja de trigo la viabilidad microbiana fue menor a los obtenidos en el presente estudio.

\section{Conclusiones y recomendaciones}

La inclusión de L. acidophillus, S. thermophillus y K. fragilis (L-4 UCLV) aumentó los valores en la materia seca, proteína cruda y verdadera, ácido láctico y disminuyó el pH en el ensilaje de yuca integral con $20 \%$ harina de caupí, por 18 meses de conservado.

Los datos del presente estudio sugieren que la adición de preparado microbiano en el ensilaje de yuca integral con caupí puede solubilizar algunas de las partes de las fibras de fácil digestión de los carbohidratos de la pared celular y, por lo tanto, puede suministrar sustratos para la fermentación de ácido láctico.

Así mismo, la adición de bacterias lácticas y levaduras en el ensilaje podría ser eficaz para mejorar la calidad y mantener sobre los 18 meses de conservado a la yuca integral (hojas, raíz, tallo) con harina de caupí, debido a la disminución en el contenido de azúcar y/o al aumento en la concentración de proteínas.

\section{Agradecimiento}

El autor principal agradece a Instituto de Fomento a Talento Humano (IFTH), Secretaría de Educación Superior, Ciencia, Tecnología e Innovación (SENESCYT), Ecuador, por la beca de formación doctoral (PhD). 


\section{Bibliografía}

Acevedo, I., García, O., Contreras, J., y Acevedo, I. (2009). "Elaboración y evaluación de las características sensoriales de un yogurt de leche caprina con jalea semifluida de piña". Revista UDO Agrícola, 9 (2), 442-448.

Ali, M., Cone, J.W., van Duinkerken, G., Klop, A., Kruisdijk, J., Blok, M.C., Bruinenberg, M., y Hendriks, W.H. (2014). "Relationship between chemical composition and in situ rumen degradation characteristics of grass silages in dairy cows". NJAS-Wageningen Journal of Life Sciences, 70,9-15.

AOAC. (2012). "Official methods of analysis, Association of official analytical chemist 19th edition", Washington D.C., USA.

Conway, E.J. (1957). Microdiffusion Analysis and Volumetric Error. 4th ed. Crosby Lockwood and Son, London, Engl.

Dadvar, P., Dayani, O., Mehdipour, M., y Morovat, M. (2015). Determination of physical characteristics, chemical composition and digestion coefficients of treated lemon pulp with Saccharomyces cerevisiae in goat diet. Journal of Animal Physiology and Animal Nutrition 99(1):107-113.

Danelon, J.L., D’Alesio, M., Barletta, L., Allocati, P.A., Wawrzkiewicz, M., Ceballos, E., Colatto, C., y Victoria, D. (2010). "Ensilaje de alfalfa suplementado con distintos concentrados energéticos para alimentar cabras lecheras". Asociación Latinoamericana de Producción Animal, 18(1-2), 17-26.

del Rio, S,. Heguy, J.M., Lago, A. (2010). "Feed management practices on California dairies". Journal of Dairy Science, 93 (Suppl.1), 773.

Der Bedrosian, M.C., Nestor, K.E., y Kung, L. (2012). "The effects of hybrid, maturity, and length of storage on the composition and nutritive value of corn silage". Journal of Dairy Science, 95:5115-5126.

Duncan, D.B. (1955). "Multiple range and multiple F tests". Biometrics 11:1.

Fernández-Herrero, A., Tabera, A., Agüeria, D., y Manca, E. (2013). "Obtención, caracterización microbiológica y físicoquímica de ensilado biológico de anchoíta (Engraulis anchoíta)". REDVET - Revista electrónica de Veterinaria, 14(2).

Guglielmo, S., Proietti, S., Moscatello, S., Stefanoni, W., y Battistelli, A. (2015). "Anaerobic digestion of corn silage on a commercial scale: Differential utilization of its chemical constituents and characterization of the solid digestate". Biomass and Bioenergy, 83, 17-22.

Heguy, J.M., Karle, B.M., Price, P.L., y Meyer, D. (2010). “Calculating field nutrient removal rates to comply with General Order for Existing Milk Cow Dairies from California's Central Valley Regional Water Quality Control Board". Journal of Dairy Science, 93 (Suppl. 1), 412.

Heguy, J.M., Meyer, D., y Silva-del-Río, N. (2016). "A survey of silage management practices on California dairies". Journal of Dairy Science, 99(2), 1-6

Hoffman, P.C., Esser, N.M., Shaver, R.D., Coblentz, W.K., Scott, M.P., Bodnar, A.L., Schmidt, R.J., y Charley, R.C. (2011). "Influence of ensiling time and inoculation on alteration of the starchprotein matrix in high-moisture corn". Journal of Dairy Science, 94, 2465-2474.

Ke, W.C., Yangb, F.Y., Undersander, D.J., y Guo, X.S. (2015). "Fermentation characteristics, aerobic stability, proteolysis and lipid composition of alfalfa silage ensiled with apple or grape pomace". Animal Feed Science and Technology, 202, $12-$ 19.

Kung, L., Lim, J.M., Hudson, D.J., Smith, J.M., y Joerger, R.D. (2015). "Chemical composition and nutritive value of corn silage harvested in the northeastern United States after Tropical Storm Irene". Journal of Dairy Science, 98(3), 2055-2062.

Lyncha, J., Jina, L., Larab, E.C., Baaha, J., y Beauchemina, K.A. (2014). "The effect of exogenous fibrolytic enzymes and a ferulic acidesterase-producing inoculant on the 
fibre degradability, chemical composition and conservation characteristics of alfalfa silage". Animal Feed Science and Technology, 193, 21-31.

Miranda, J.E., Marin, A., y Baño, D. (2017). "Elaboration of a bioprepared with probiotic effect from a mixed culture of lactic bacteria and yeasts". Bionatura, 2(1), 245-247.

Miranda-Yuquilema, J.E., Marin-Cárdenas, A., y Gonzales-Pérez, M. (2014). "Selage of cassava (Manihot esculenta Crantz) with cowpeas (Vigna unguiculata) for pig alimentation". REDVET - Revista electrónica de Veterinaria. 15(2).

Sourav, B., y Arijit, D. (2010). "Study of physical and culture parameters on the bacteriocins produced by lactic acid bacteria isolated from traditional indian fermented food". American Journal of Food Technology, 5 (2), 111-120.

Spanghero, M., Zanfi, C., Signor, M., Davanzo, D., Volpe, V., y Venerus, S. (2015). "Effects of plant vegetative stage and fielddrying time on chemical composition and in vitro ruminal degradation of forage soybean silage". Animal Feed Science and Technology, 200,102-106. 